\title{
Ensayo LE CHATELIER-ANSTETT
}

\author{
JOSE LUIS SAGRERA \\ Lcdo. en Ciencias Químicas \\ Jefe de la Sección de Corrosión del Hormigón \\ Instituto Eduardo Torroja de la Construcción y del Cemento
}

\section{INTRODUCCION}

Uno de los procedimientos de estudio de la sulfatodurabilidad de los conglomerantes hidráulicos, es el ensayo de Le Chatelier-Anstett (1).

Dicho ensayo, tiene por objeto, en líneas generales, comprobar el comportamiento de los cementos hidratados y amasados con yeso dihidrato, al cabo de 28 días en cámara húmeda.

La División de Durabilidad de este Instituto, desde hace más de un año, lleva a cabo el estudio de dicho ensayo, empleando diferentes normas de preparación de probetas, sometidas cada una de ellas a dos diferentes agresivos $\left(\mathrm{SO}_{4} \mathrm{Ca} \cdot 2 \mathrm{H}_{2} \mathrm{O}\right.$ y $\left.\mathrm{SO}_{4} \mathrm{Na}_{2} \cdot 10 \mathrm{H}_{2} \mathrm{O}\right)$.

Los resultados hallados van acompañados de los obtenidos, aplicando la técnica de análisis cualitativo por difracción de rayos $\mathrm{X}$.

Con objeto de que cada muestra evolucione independientemente de las restantes, se han

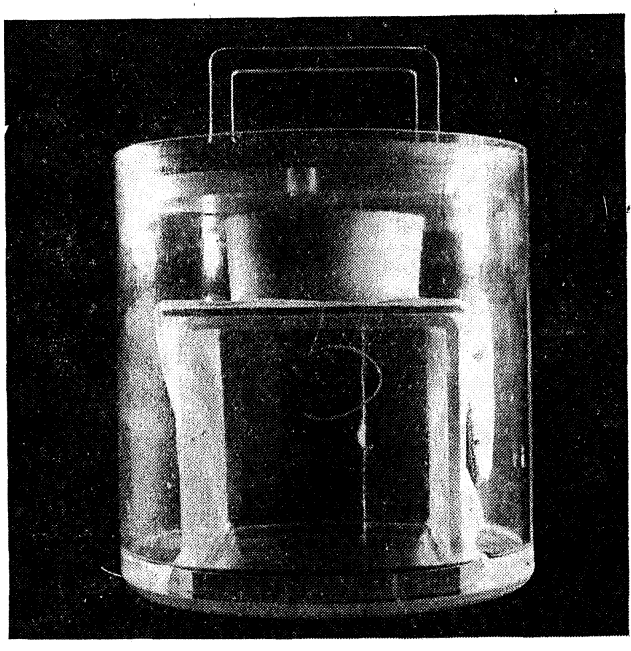

Foto 1 alojado en sendos recipientes de plástico, herméticamente cerrados con una tapa del mismo material (foto 1 ).

Las fechas de ensayo para cada grupo de probetas son 7, 28 y 90 días prolongándose hasta 180 días, transcurridos los cuales, y para cada norma y agresivo, se llevará a cabo una publicación con los resultados obtenidos $\mathrm{y}$ las conclusiones posibles.

\section{CEMENTOS ESTUDIADOS}

Los cementos tipo ensayados, con objeto de hacer lo más extenso posible el estudio, abarcan desde un portland ordinario hasta un portland de alto horno, pasando por tres resistentes a los sulfatos y dos puzolánicos. 
Dentro de nuestros ensayos han sido clasificados con la numeración siguiente:

\begin{tabular}{cll} 
Cemento & & \multicolumn{1}{c}{ Clase } \\
\cline { 1 - 1 } $\mathrm{N}^{\circ} 1$ & & Cemento portland ordinario \\
$\mathrm{N}^{\circ} 2$ & & Cemento PAS - I \\
$\mathrm{N}^{\circ} 3$ & & Cemento Puzolánico \\
$\mathrm{N}^{\circ} 4$ & & Cemento PAS-Puzolánico \\
$\mathrm{N}^{\circ} 5$ & & Cemento Portland de alto horno \\
$\mathrm{N}^{\circ} 6$ & & Cemento PAS-A \\
$\mathrm{N}^{\circ} 7$ & & Cemento PAS-E
\end{tabular}

Los análisis químicos de cada uno de ellos son:

Análisis químico de los cementos

\begin{tabular}{|c|c|c|c|c|c|c|c|}
\hline $\begin{array}{c}\text { Siglas del } \\
\text { CEMBUREAU }\end{array}$ & o.P.C. & S.R.P.C. & P.C.z. & $\begin{array}{c}\text { P.c.z. } \\
\text { sp. }\end{array}$ & B.L.C. & S.R.P.C. & S.R.P.C. \\
\hline Numeración & $\mathbf{1}$ & 2 & 3 & 4 & 5 & 6 & 7 \\
\hline $\mathrm{SiO}_{2}$ & 20,87 & 21,21 & 19,73 & 31,90 & 29,30 & 19,53 & 20,8 \\
\hline R.I. & 0,10 & 0,04 & 13,60 & 5,08 & 0,38 & - & - \\
\hline $\mathrm{Al}_{2} \mathrm{O}_{3}$ & 5,24 & 3,75 & 9,99 & 3,02 & 11,70 & 4,55 & 3,7 \\
\hline $\mathrm{Fe}_{2} \mathrm{O}_{3}$ & 2,80 & 4,13 & 3,45 & 3,13 & 1,04 & 6,60 & 5,4 \\
\hline $\mathrm{CaO}$ & 63,87 & 64,44 & 43,51 & 47,94 & 45,85 & 65,50 & 62,8 \\
\hline MgO & 1,36 & 1,14 & 1,37 & 0,88 & 4,91 & 0,70 & 1,4 \\
\hline $\mathrm{SO}_{3}$ & 3,45 & 2,74 & 1,95 & 2,99 & 2,83 & 2,60 & 1,5 \\
\hline P.F. & 1,10 & 1,32 & 3,13 & 4,39 & 0,95 & 0,40 & 2,5 \\
\hline $\mathrm{K}_{2} \mathrm{O}$ & 0,86 & 0,94 & 2,46 & 0,71 & 0,88 & - & - \\
\hline $\mathrm{Na}_{2} \mathrm{O}$ & 0,37 & 0,21 & 0,69 & 0,16 & 0,74 & - & - \\
\hline
\end{tabular}

\section{NORMAS DE PREPARACION DE PROBETAS}

Se han empleado dos normas, una de ellas con dos variantes, lo cual hace que se consideren como tres normas diferentes en nuestro ensayo.

\subsection{Norma francesa (200)}

Es la adoptada por L. Blondiau (1) para este ensayo con las siguientes especificaciones:

2.1.1. El cemento anhidro se muele hasta pasar por tamiz de 0,088 milímetros.

2.1.2. Dos partes de cemento se amasan con una de agua.

2.1.3. La mezcla se mantiene en humedad saturada durante 24 horas. 
2.1.4. Se trocea y se sumerge bajo agua durante 14 días. ${ }^{-3} n^{-}$nuestro ensayb se 'dejó 200 días para tener seguridad.

2.1.5. Se comprueba la hidratación; para ello, la diferencia entre el peso específico del cemento anhidro y el cemento hidratado será mayor que un $20 \%$ del peso específico del cemento anhidro.

2.1.6. El cemento hidratado se trocea a tamaño de 5 milímetros.

2.1.7. Se deseca en estufa a $40^{\circ} \mathrm{C}$ hasta que dos pesadas a intervalos de 30 minutos no difieran en un $3 \%$.

2.1.8. Dos partes de cemento hidratado se mezclan con una parte de yeso dihidrato precipitado químicamente puro.

2.1.9. La mezcla se pulveriza hasta pasar por tamiz de $0,2 \mathrm{~mm}$, pero cuidando no pasar de $40^{\circ} \mathrm{C}$, para no deshidratar el yeso dihidrato.

2.1.10. A la mezcla se le añade un $6 \%$ ponderal de agua y se hace la probeta cilíndrica presionando a 4 kilopondios.

\subsection{Norma holandesa ( $\mathrm{N}-1591$, junio 1953)}

Las condiciones de preparación de probetas son:

2.2.1. Se mezclan dos partes de cemento con una parte de agua en peso.

2.2.2. Con la mezcla se preparan cubos de $7,1 \mathrm{~cm}$ de arista y se dejan 24 horas.

2.2.3. Se desenmolda el cubo y se sumerge en agua durante 14 días.

2.2.4. Se toma escayola y se amasa con agua hasta obtener una pasta fluida.

2.2.5. La pasta de escayola se vierte en molde cúbico de $7,1 \mathrm{~cm}$ de arista y se deja 2 horas.

2.2.6. Se desenmolda el cubo, se lleva a cámara con $80 \%$ de humedad y se deja 14 días.

2.2.7. Los cubos de escayola y cemento se muelen reposadamente hasta pasar por ta$\mathrm{miz}$ de $2,79 \mathrm{~mm}$, secando luego a $45^{\circ}-50^{\circ} \mathrm{C}$.

2.2.8. Se mezclan dos partes de cemento con una parte de escayola.

2.2.9. La mezcla se muele hasta pasar por tamiz de 0,2 milímetros.

2.2.10. A la mezcla se le añade un $6 \%$ de agua en peso y se prepara la probeta cilíndrica presionando a 20 kilopondios.

\subsection{Norma holandesa (200)}

Con objeto de poder comparar las normas francesa y holandesa, el tiempo de hidratación de los cubos fue de 200 días, ya que, como se recordará, al preparar las probetas según norma francesa, con objeto de asegurar la hidratación, ya que no se consideraba seguro su método de comprobación por diferencia de pesos específicos del cemento anhidro e hidratado. También se utilizó un tiempo de hidratación de 200 días.

Esta es la única variante de la norma holandesa que se ha llevado a cabo en los ensayos de la División de Durabilidad. 


\section{AGRESIVOS EMPLEADOS}

Los agresivos empleados para las probetas hechas con cada una de las tres normas, son: sulfato cálcico dihidrato y sulfato sódico decahidrato.

Como se recordará en la norma holandesa, el sulfato cálcico dihidrato se obtenía por hidratación de sulfato cálcico hemihidrato; por tanto, las materias primas para la obtención de los agresivos son: sulfato cálcico hemihidrato, sulfato cálcico dihidrato y sulfato sódico decahidrato. Cada uno de ellos se analizó cualitativamente, con técnica de difracción de rayos $\mathrm{X}$, para comprobar su pureza. A la vista de los tres diagramas se confirmó que la pureza de las materias primas era la necesaria.

\section{RECIPIENTES EMPLEADOS}

Cada una de las probetas cilíndricas se conserva en un recipiente cilíndrico de plástico transparente. La probeta se coloca sobre un puente, también de plástico, cuya parte horizontal dista $10 \mathrm{~cm}$ de la capa de agua destilada. Entre la probeta y la parte horizontal del puente, existe una hoja de papel de filtro cuyos extremos están sumergidos en el agua destilada que contiene el recipiente. Dicho recipiente y para preservar, tanto la probeta como el agua destilada, de posibles contaminaciones exteriores, va cerrado con una tapa del mismo material. En la tapa se ha practicado un orificio que cierra mediante tapón de goma, para, a cada edad del ensayo, hacer toma de $25 \mathrm{~mm}$ de líquido y en su día realizar análisis químicos para determinar el posible aporte de productos de la probeta al agua destilada. Con objeto de no disminuir la cantidad de líquidos dentro del recipiente, se rellena con igual cantidad $(25 \mathrm{ml})$ de agua destilada. Toda la descripción anterior puede comprenderse más fácilmente consultando la foto 1 .

El ajuste de tapa y cilindro se realiza gracias a una junta circular de plástico flexible que rodea el borde circular de la tapa.

Los recipientes van duplicados para alojar un número doble de probetas: una para ensayo de difracción de rayos $\mathrm{X}$ y otra testigo para hacer medidas de su diámetro y altura.

\section{ENSAYOS REALIZADOS}

Cada una de las probetas, y en su fecha correspondiente a los 7, 28, 90 y 180 días de permanencia en su recipiente, se extrae del mismo. Sobre la tomada como testigo se mide diámetro y altura, así como se comprueba también su dureza mediante la aguja de Vicat. Sobre la probeta duplicada, alojada en otro recipiente de plástico, se lleva a cabo mediante un taladra-corchos, 'si su dureza lo permite, en caso contrario con una broca especial, según foto 2, la toma de un testigo cilíndrico de igual altura que la probeta.

De este testigo, conservado en cámara húmeda, se toman cuatro lajas, por corte perpendicular a la generatriz del cilindro (foto 3 ), que corresponderán: la laja $\mathrm{n}^{\circ} 1$, a la parte inferior de la probeta; las lajas $\mathrm{n}^{\mathrm{os}} 2$ y 3 , a la parte central inferior y superior, respectivamente, y finalmente, una laja $\mathrm{n}^{\circ} 4$, que corresponde a la parte superior de la probeta. Cada una de estas lajas, y por separado, se analiza por difracción de rayos X, para detectar de una manera "semicuantitativa" la cantidad que de cada compuesto "cristalino" tiene la laja representativa de la porción considerada de cada probeta.

\section{CONDICIONES OPERATORIAS DEL EQUIPO DE RAYOS $\mathbf{X}$}

Los análisis de difracción de rayos $\mathrm{X}$ han sido realizados con un equipo marca Philips, tipo manual de $1 \mathrm{~kW}$. 


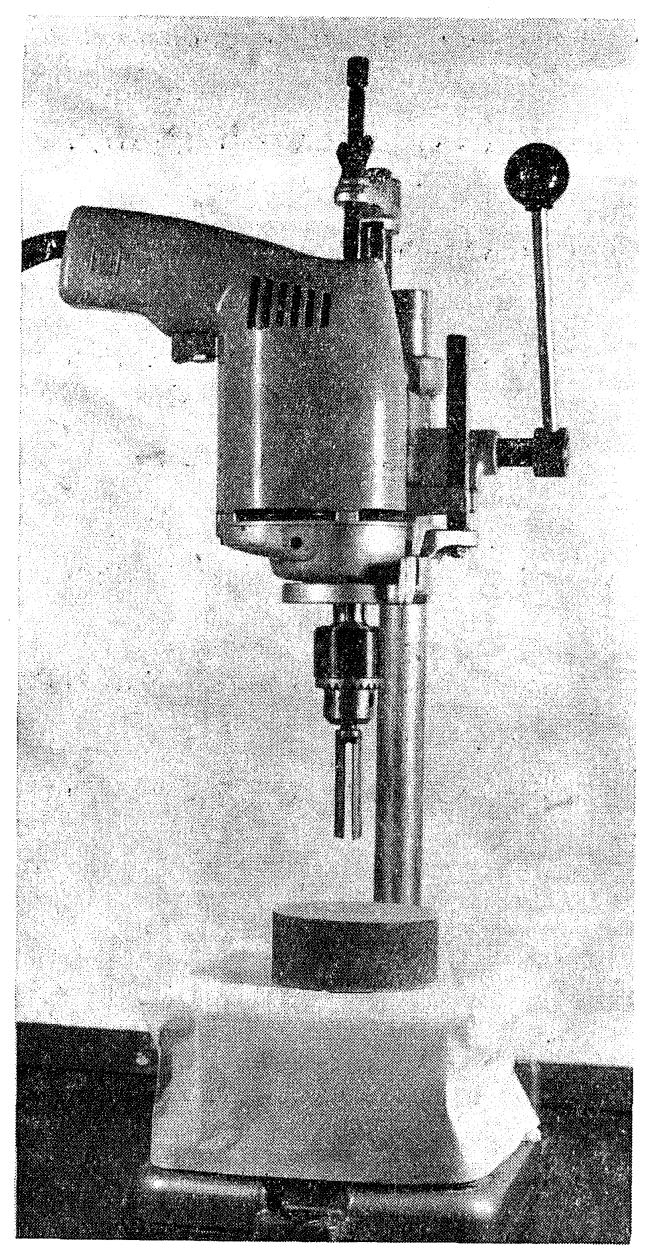

Foto 2

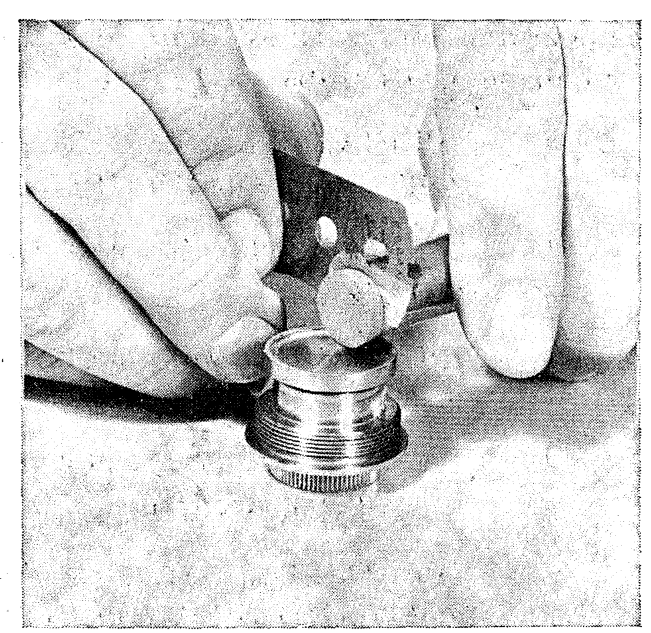

Foto 3

Las condiciones operatorias del equipo son:

- Anodo de cobre: trabajando a $40 \mathrm{kV} \mathrm{y} 24 \mathrm{~mA}$.

- Rendijas de colimación: $1^{\circ}-0,2-1^{\circ}$.

- Velocidad de goniómetro: $2^{\circ}$ por minuto.

- Factores de escala: 8-2-1.

- Velocidad de registro: 40.

- Tensión del contador proporcional: $1.650 \mathrm{~V}$.

Sobre cada gráfica obtenida de cada laja analizada se determinan "semicuantitativamente" las concentraciones, midiendo las alturas de cada pico representativo. Los datos obtenidos son llevados a un cuadro general de resultados, para luego por comparación, y teniendo en cuenta las variables del ensayo, poder deducir las conclusiones pertinentes. Las gráficas de cada análisis de rayos $\mathrm{X}$, dada su superficie y cantidad, no figuran en el presente trabajo, quedando archivados con la copia del mismo para posible consulta futura.

\section{CONCLUSIONES}

Dado que el número de cementos estudiados es de siete, que las normas de preparación de probetas son tres y que el número de agresivos es de dos, las variables totales del presente estudio son:

$$
7 \times 3 \times 2=42 \text { variables. }
$$

Como los resultados son muchos y las conclusiones varias, se ha pensado resumir en una 
monografía los ensayos realizados con cada uno de los siete cementos empleados. De esta manera, los trabajos futuros serán:

\author{
I - APLICACION DEL METODO LE CHATELIER-ANSTETT A UN CEMENTO \\ PORTLAND ORDINARIO \\ II - APLICACION DEL METODO LE CHATELIER-ANSTETT A UN CEMENTO \\ PAS (I) \\ III - APLICACION DEL METODO LE CHATELIER-ANSTETT A UN CEMENTO \\ PUZOLANICO \\ IV - APLICACION DEL METODO LE CHATELIER-ANSTETT A UN CEMENTO \\ PAS PUZOLANICO \\ V - APLICACION DEL METODO LE CHATELIER-ANSTETT A UN CEMENTO \\ PORTLAND DE ALTO HORNO \\ VI - APLICACION DEL METODO LE CHATELIER-ANSTETT A UN CEMENTO \\ PAS (A) \\ VII - APLICACION DEL METODO LE CHATELIER-ANSTETT A UN CEMENTO \\ PAS (E)
}

\title{
B I B L I O G R A F I A
}

(1) Blondiau, L.: "Consideration diverses relatives a l'essai de résistance chimique au sulfate de calcium suivant le processus Le Chatelier-Anstett". Rev. Mat. de Construction. Mars. 1961, n. ${ }^{\circ}$ 546, pp. 189-200.

JASPERS, M. J. M.: “Contribution a l'etude experimentale de la mesure par l'essai Le Chatelier-Anstett de la résistance des ciments aux sulfates et chlorures". Ciments et Bétons 633-634. Juin 1968.

Garcia de Faredes, P.: "Monografía n..$^{232 "}$ del Instituto Eduardo Torroja de la Construcción y del Cemento. Madrid 1963.

Garcia de Paredes, P. y SAgrera J. L.: "Cuaderno de Investigación n.o 25" del Instituto Eduardo Torroja de la Construcción y del Cemento. Noviembre 1969. 